\title{
Epileptischer Anfall
}

\author{
J. Rémi, S. Noachtar
}

\section{Ein 19-jähriger Mann wird nach einem erstmals aufgetretenen „Krampf- anfall“ vorgestellt. Nach einer Party sei er plötzlich nicht mehr kontakt- fähig gewesen und habe für ein bis zwei Minuten am ganzen Körper gekrampft. In der Nothilfe ist er fokal-neurologisch unauffällig.}

_ Im Anfall sollte der Patient vor weiteren Verletzungen geschützt werden. Sinnvoll sind die stabile Seitenlage und das Entfernen gefährlicher Gegenstände aus dem Umfeld. Epileptische Anfälle sind typischerweise selbstlimitierend und dauern selten länger als 90 Sek. Die postiktale Phase dauert oft deutlich länger. Deshalb werden prähospitale Anfälle oft nur von Laien beobachtet, was bei der Schilderung der Semiologie und Dauer (hier meist Überschätzung) bedacht werden muss.

\section{Differenzialdiagnose}

Neben epileptischen Anfällen sind Gelegenheitsanfälle, konvulsive Synkopen und psychogene Anfälle zu bedenken. Gelegenheitsanfälle treten nur im Zusammenhang mit provozierenden Faktoren (Alkoholentzug) auf und sistie-

\section{Kasuistik}

\section{WIE GING ES WEITER?}

Nach mehrmaligem Nachfragen berichtet der Patient über morgendliche Zuckungen (Myoklonien) der Arme seit zwei Jahren. Im EEG werden Polyspike-Wave-Komplexe aufgezeichnet. Es wird eine juvenile-myoklonische Epilepsie diagnostiziert. Unter Therapie mit Levetiracetam wird der Patient anfallsfrei. Bei diesem Syndrom muss wegen eines hohen Rückfallrisikos das Medikament ein Leben lang eingenommen werden. ren, wenn diese beendet werden. Bei manchen Epilepsien kommen solche Faktoren ebenfalls als Auslöser in Frage.

Unser Patient hatte zusätzliche generalisierte myoklonische Anfälle (s. Kasten). Daher ist bei ihm nicht von einem Gelegenheitsanfall auszugehen. Hilfreich war hier auch das EEG, das nach einem ersten Anfall immer sinnvoll ist. Werden nach einem Anfall epilepsietypische Potenziale nachgewiesen, besteht eine Epilepsie. Ein normales EEG schließt eine Epilepsie dagegen nicht aus.

Synkopen gehen insbesondere bei jungen Patienten sehr oft mit motorischen Entäußerungen (tonisch-klonisch) einher, die für den Laien nicht gut von epileptischen Kloni zu unterscheiden sind. Typischerweise sind diese Patienten jedoch rasch reorientiert. Hier sollte eine internistische Diagnostik folgen.

Psychogene Anfälle zeichnen sich durch längere Dauer, An- und Abschwellen der Symptomatik und Auftreten oft unter Anwesenheit von Zeugen aus. Die Differenzialdiagnose (DD) kann nach einem ersten Anfall schwierig sein. Wenn Anfälle häufig genug auftreten, ist ein EEG-Video-Monitoring zur DD indiziert.

Der Notarzt sollte akute Ursachen wie Hypoglykämien oder eine Hypotonie ausschließen.

\section{Therapie}

Die Gabe von Benzodiazepinen im Anfall kann aus pharmakokinetischer Sicht den akuten Anfall mit einer Dauer von einer Minute nicht beenden. Benzodiazepine (bevorzugt Lorazepam, Midazolam oder Clonazepam) können jedoch sinnvoll sein, um einen Status epilepticus oder wiederholte Anfälle zu vermeiden, insbesondere in Situationen, in denen der Notarzt wenig Informationen zum Patienten hat. Besteht eine Epilepsie, sollte bereits nach dem ersten Anfall eine Therapie mit einem passenden Antiepileptikum begonnen werden (www. dgn.org/leitlinien).

\section{An was noch denken?}

Bei jeder Art von Anfall (auch bei nichtepileptischen Anfällen!) muss eine dokumentierte Führerscheinberatung erfolgen. Hier sind die Leitlinien des Bundesamts für Straßenwesen hilfreich (www. bast.de). Zudem sollten allgemeine Gefährdungssituationen (Schwimmen, Klettern etc.) angesprochen werden. Eine soziale Beratung bei Epilepsie wird durch Beratungsstellen unterstützt (www.epilepsie-online.de).

\footnotetext{
$\rightarrow$ Für die Verfasser: PD Dr. med. Jan Rémi Epilepsie-Zentrum Neurologische Klinik und Poliklinik Klinikum der Universität München Marchioninistraße 15, D-81377 München E-Mail: Jan.Remi@med.uni-muenchen.de

$\rightarrow$ Koautor: Prof. Dr. med. Soheyl Noachtar, München
} 